\title{
The 2003 eclipse of EE Cep is coming
}

\section{A review of past eclipses}

\author{
D. Graczyk ${ }^{1}$, M. Mikołajewski ${ }^{1}$, T. Tomov ${ }^{1}$, D. Kolev², and I. Iliev² \\ 1 Centre for Astronomy, ul. Gagarina 11, 87-100 Toruń, Poland \\ e-mail: weganin@astri.uni.torun.pl; mamiko@astri.uni.torun.pl \\ 2 National Astronomical Observatory Rozeh, Institute of Astronomy, BAS, PO Box 136, 4700 Smolyan, Bulgaria
}

Received 18 December 2002 / Accepted 17 March 2003

\begin{abstract}
EE Cep is an eclipsing binary with a period of 5.6 years. The next eclipse will occur soon, in May-June 2003, and all available past eclipses were collected and briefly analysed. EE Cep shows very large changes of the shape and the depth of minima during different eclipses, however it is possible to single out some persistent features. The analysis suggests that the eclipsing body should be a long object surrounded by an extended semi-transparent envelope. As an explanation, a model of a precessing optically thick disc, inclined to the plane of the binary orbit, is invoked. The changes of its spatial orientation, which is defined by the inclination of the disc and the tilt, induced most probably by precession of the disc spin axis with a period of about 50 years, produce strange photometric behaviour of this star. The $\mathrm{H} \alpha$ emission, and possibly the NaI absorptions, show significant changes during several months outside of the eclipse phase.
\end{abstract}

Key words. binaries: eclipsing - stars: individual: EE Cep

\section{Introduction}

EE Cep $\left(\mathrm{BD}+55^{\circ} 2693\right)$ is an extraordinary long-period eclipsing binary showing very large and complex changes of the shape and the depth of the minima (e.g. Baldinelli \& Ghedini 1977). Quite recently Mikołajewski \& Graczyk (1999, hereafter MG) have suggested that the reason for these changes is the presence of a non-stellar eclipsing body in the system a precessing, opaque and dark disc.

This star was identified as a variable star by Romano (1956) in 1952 and it was confirmed by Weber (1956) who reported observations of the previous minimum in 1947. The colour changes during the 1952 minimum were very small and the star was originally classified by Romano as an $\mathrm{R} \mathrm{CrB}$ variable. Perova (1957) reported about 500 photographic observations of EE Cep between 1899-1908 and 1933-1955 but no brightness changes were recorded. Romano \& Perissinotto (1966) have suggested that it may be an eclipsing binary but they did not give any approximate ephemeris. Its binary nature was confirmed later by Meinunger (1973) who analysed over 500 photographic plates from Sonneberg Observatory. The period of the star is almost 2050 days and only the primary minimum can be detected. Meinunger has not detected any changes of the brightness between the 1958 and 1964 minima. The brightness of the star outside of the eclipse phases is constant within the

Send offprint requests to: D. Graczyk,

e-mail: darek.graczyk@astri.uni.torun.pl observational errors: $V_{\max }=10.78$ (Meinunger \& Pfau 1981, MG). The primary component is apparently a normal B5 III star (Herbig 1960; Baldinelli et al. 1981). The emission visible in $\mathrm{H}_{\alpha}$ and $\mathrm{H}_{\beta}$ lines arises most likely from an invisible companion (Brïckner 1976). The absorption spectrum of the companion is never seen.

The early model of EE Cep presented by Meinunger (1976) assumed that the companion should be a cool star of $\mathrm{M}$ type, much less luminous but larger than the primary B5 III star, surrounded by a strongly extended envelope. The pulsations of the cool star would change its diameter and thus lead to the changes of the minima duration and depth. The envelope would cause an atmospheric eclipse in the beginning and in the end of the minimum, producing typical wings in the light curves. However, such a simple model cannot explain some of the features observed in EE Cep, as was argued by MG and in this paper.

\section{Photometric behaviour}

\subsection{A review of past eclipses}

The typical minimum of EE Cep has large wings, the duration of the eclipse is $D \approx 40$ days or a phase interval $\Delta P=0.02$, and the depth of the eclipse is moderate $\Delta B \approx 1.5 \mathrm{mag}$. Such minima were observed in 1975, 1980 and 1997. But some eclipses observed up to date deviated strongly from this picture. We have collected observations of all previously observed eclipses of EE Cep in two roughly similar photometric 

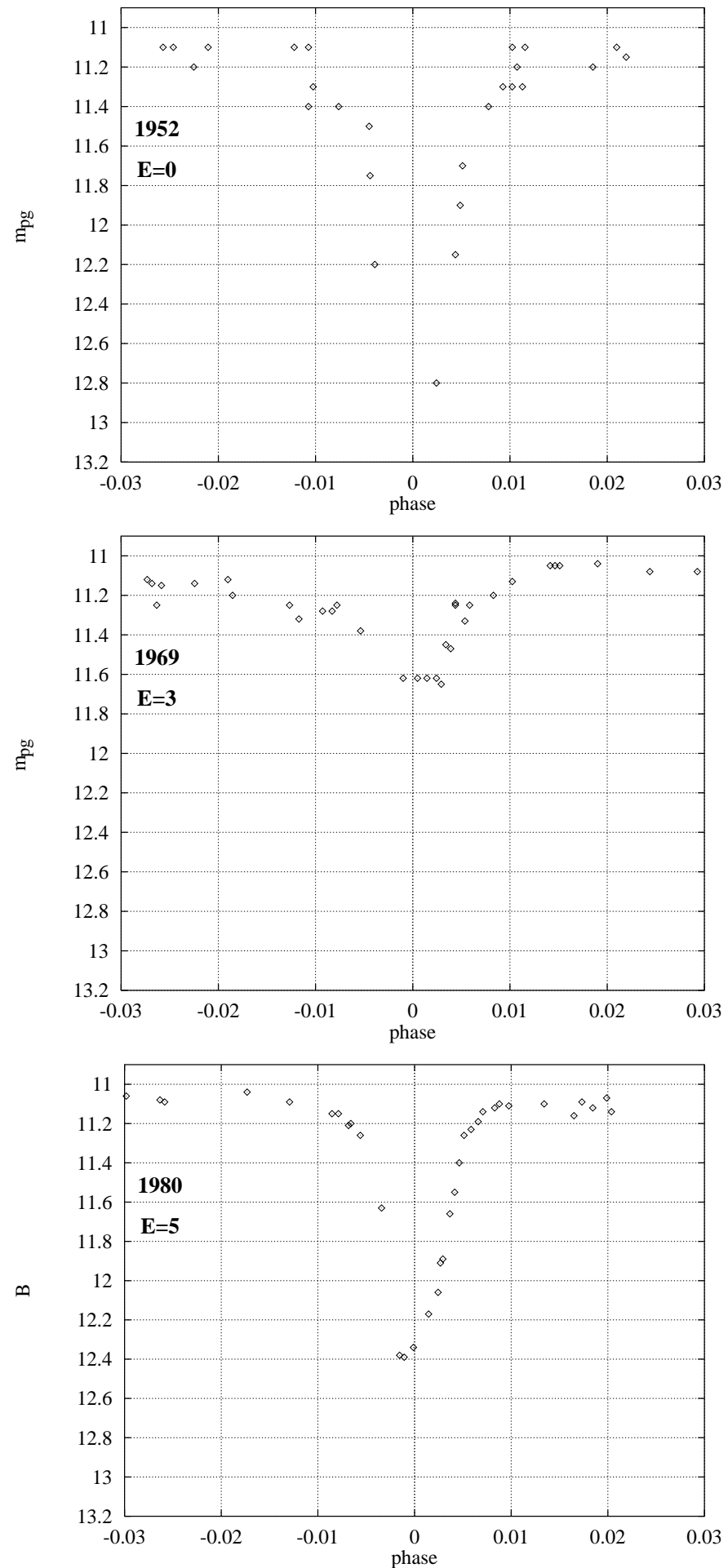

Fig. 1. EE Cep's eclipses in $B / m_{\mathrm{pg}}$ light.

wavelength bands: 1) $B$ and $m_{\mathrm{pg}}$; 2) $V, m_{\mathrm{pv}}$ and $m_{\mathrm{vis}}$. The references used for the photometry are listed in Table1, whereas the data are presented in Figs. 1 and 2. Part of the historical data were published only as diagrams and they needed to be read from these figures. All observations were phased using the ephemeris given by MG.

The 1947 minimum $(E=-1)$ was the first one detected and its depth was 1.5 in $m_{\mathrm{pg}}$. There is no information about the
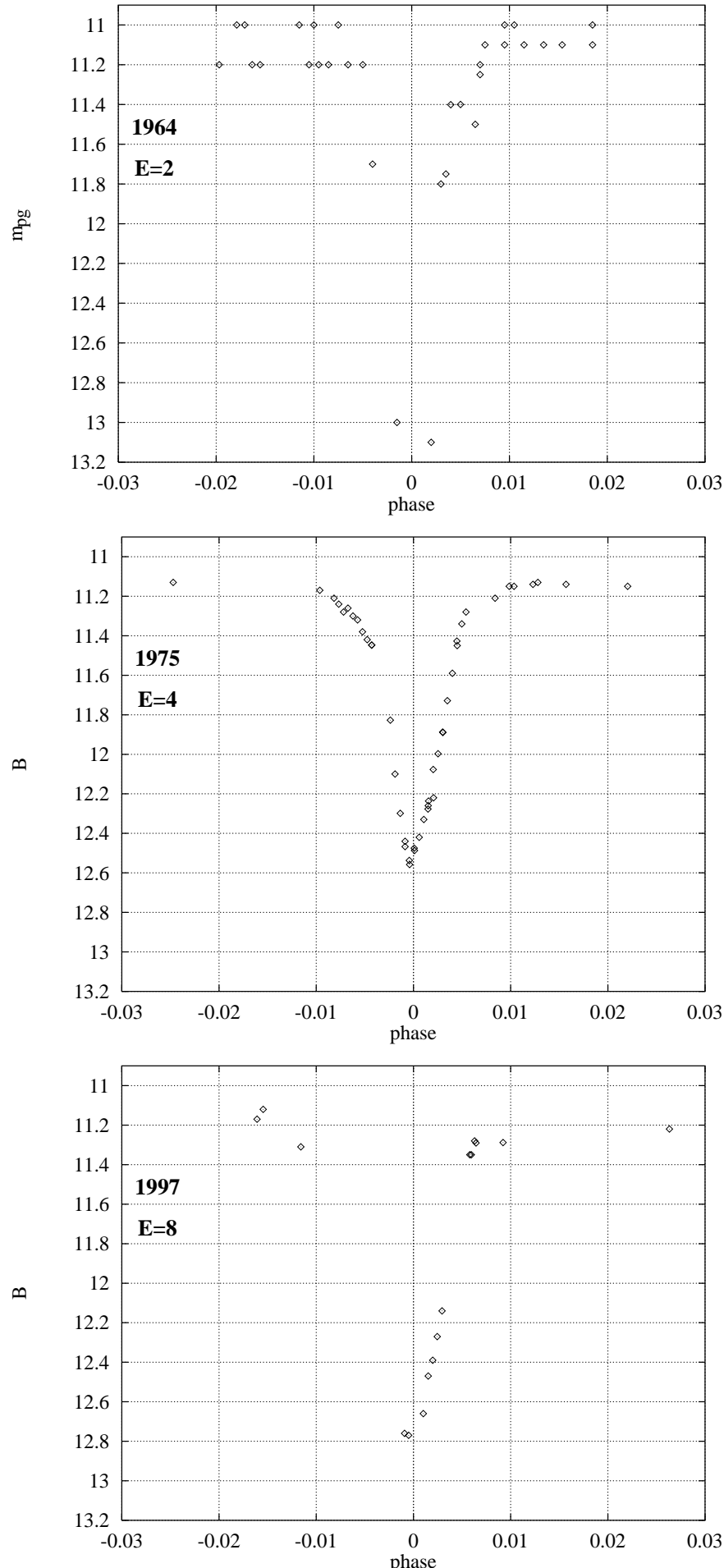

duration and shape of the eclipse and it is not shown in Fig. 1. The 1952 minimum $(E=0)$ was deep (at least 1.7 mag in $m_{\mathrm{pg}}$ ) and has an asymmetric shape. During the next eclipse, in 1958 $(E=1)$, there are only two observational points and two upper limits $\approx 13$. in $m_{\mathrm{pg}}$ at the bottom of the minimum (Romano \& Perissinotto 1966), therefore we also omitted this minimum in Fig. 1. Nevertheless, it could be very similar, or maybe a little deeper, to the following one in $1964(E=2)$. The depth of 

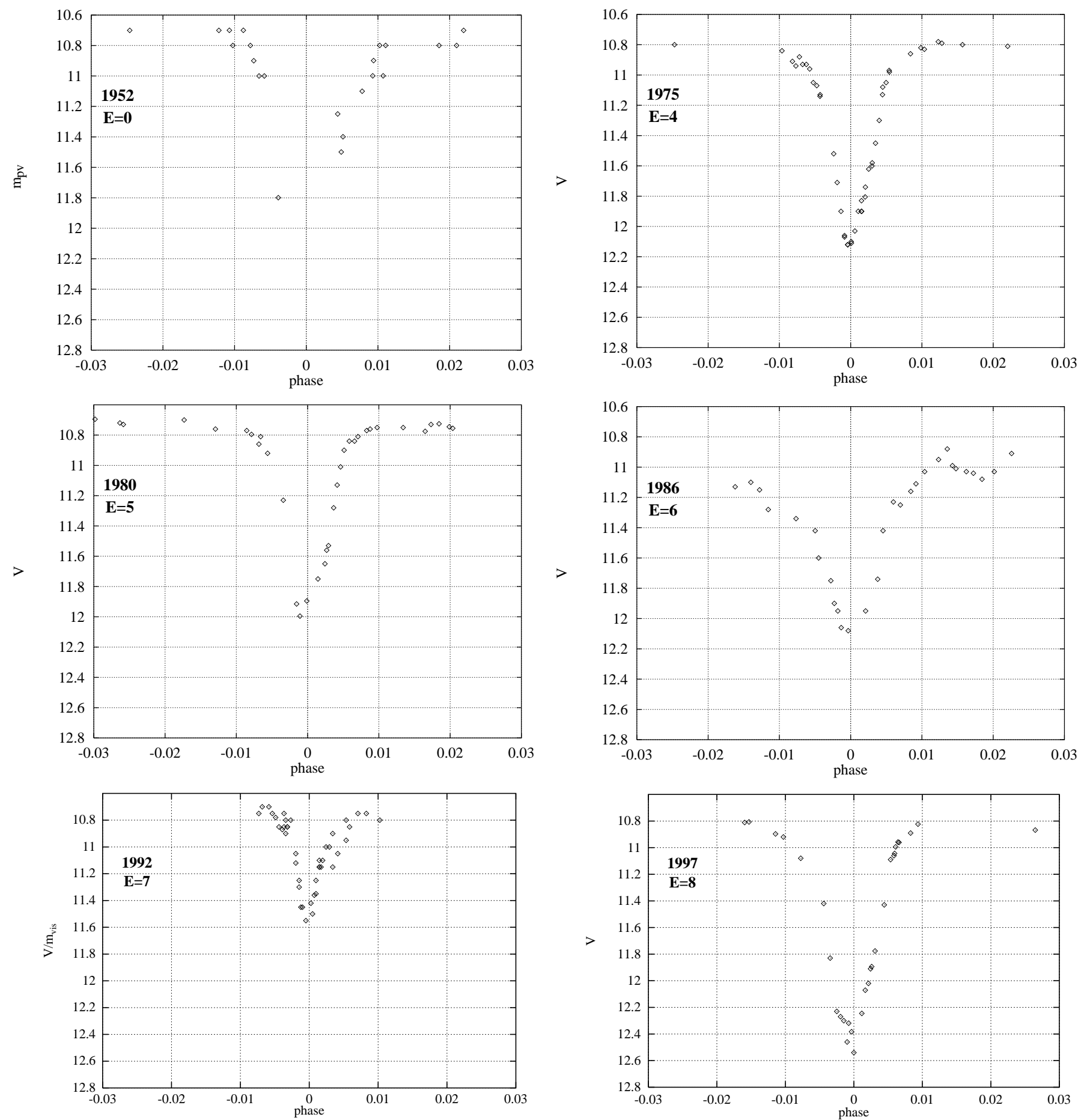

Fig. 2. EE Cep's eclipses in $V / m_{\mathrm{pv}} / m_{\mathrm{vis}}$ light.

these two minima is at least 2 mag. The duration of the 1964 eclipse was only 30 days.

During the $1969(E=3)$ eclipse, the minimum was, in contrast to the previous one $(E=2)$, extremely shallow $\left(\Delta m_{\mathrm{pg}}=0.6 \mathrm{mag}\right)$ and a flat bottom phase was clearly observed. Also, the 1969 eclipse was probably the longest one - its total duration was about 60 days. The $1975(E=4)$ and $1980(E=5)$ eclipses were more or less typical, while the $1986(E=6)$ minimum was again shallow and strongly asymmetric. The $1992(E=7)$ eclipse was observed visually by

three amateur astronomers from AAVSO and seven $V R$ measurements with a CCD camera have been obtained by J. Borovicka from Ondrejov Observatory in the Czech Republic (Halbach 1992). Unfortunately, there is a zero-point offset of $+0{ }^{\mathrm{m}} 16$ between Borovicka's $V$ magnitude scale and Meinungers's (1976) and MG's scales. Also, the visual light curves strongly suffer from differences in zero-point scales. Thus we have shifted visual estimations to the corrected CCD observations, although three points still lie out of the light curve (Fig. 2). This minimum was shallow $\left(\Delta m_{\mathrm{vis}} \approx 0.8\right)$ and 
Table 1. The duration and the depth of eclipses.

\begin{tabular}{cccccl}
\hline \hline Epoch & Year & $\Delta P$ & $\Delta V / m_{\mathrm{pv}} / m_{\mathrm{vis}}$ & $\Delta B / m_{\mathrm{pg}}$ & References [source of data] \\
\hline-1 & 1947 & - & - & $1.5:$ & Weber (1956) [table] \\
0 & 1952 & $0.020 \pm 0.002$ & $>1.2$ & $1.7 \pm 0.2$ & Romano (1956) [figure] \\
1 & 1958 & $0.015 \pm 0.003$ & - & $1.9 \pm 0.2$ & Romano \& Perissinotto (1966) [table] \\
2 & 1964 & $0.015 \pm 0.002$ & - & $2.1 \pm 0.2$ & Meinunger (1973) [figure] \\
3 & 1969 & $0.030 \pm 0.003$ & - & $0.6 \pm 0.1$ & Baldinelli et al. (1975) [table] \\
4 & 1975 & $0.021 \pm 0.001$ & $1.34 \pm 0.02$ & $1.43 \pm 0.02$ & Meinunger (1976) [table], Zaitseva et al. (1975) [table] \\
5 & 1980 & $0.019 \pm 0.001$ & $1.25 \pm 0.02$ & $1.30 \pm 0.02$ & Baldinelli et al. (1981) [figure], Meinunger \& Pfau (1981) [table] \\
6 & 1986 & $0.025 \pm 0.002$ & $1.1 \pm 0.1$ & - & Di Luca (1988) [figure] \\
7 & 1992 & $0.011 \pm 0.002$ & $0.85 \pm 0.15$ & - & Halbach (1992) [figure] \\
8 & 1997 & $0.023 \pm 0.001$ & $1.61 \pm 0.02$ & $1.68 \pm 0.02$ & MG, Halbach (1999) [figure] \\
\hline
\end{tabular}

Note: the total duration was calculated as time period between 1st and $4^{\dagger}$ th external moments (see Sect. 2.2) and have been expressed as a part of the orbital period $(\Delta P)$. Errors were estimated according to the accuracy of the data and the quality of the light curve covering.

probably the shortest one - its total duration was about 22 days. The last observed $1997(E=8)$ eclipse was, in contrary, deeper than usual. However, its shape and the total duration were similar to those of 1975 and 1980.

\subsection{The characteristic phases of the minimum}

In general we can note that 1) the shape of the eclipse may change from the deep and narrow minimum (like in 1964) to a shallow and very wide minimum $(1969,1986), 2)$ a flat-bottom phase may occur which is characteristic for annular eclipses, 3) wide wings are often visible and 4) there is a persistent asymmetry of the light curve between the descending and the ascending branches of the minima.

Although the light curve of EE Cep shows these changes, it is possible to single out the most typical shape of the minimum. MG suggested that two eclipses from 1975 and 1980 are most typical. Basing on the data from these two eclipses they construct the representative ("template") eclipse shown in Fig. 3. There are some typical features of the minimum which are also observed in other eclipses of EE Cep. The most important is the presence of six characteristic moments during an eclipse. Moments 1st, 2nd, 3rd and 4th correspond to contacts observed in normal eclipsing binaries, while moments 1st and $4^{+}$th denote the start and the end of the phase of semiatmospheric eclipse, respectively. Another feature is the asymmetry of the light curve between the ascending and descending branches of the minimum. Such asymmetry is clearly visible in eclipses of epochs 4, 5 and 8 and may be supposed to occur in the eclipse of epoch 6 . The asymmetry seems to be caused by the presence of the sloping-bottom phase (between 2nd and 3rd contacts - Fig. 3) which influences the shape of the ascending branch. This picture is supported by the fact that during eclipse of epoch 3 four distinct contacts were observed and the bottom was flat. Thus the part of the light curve between the 2nd and 3rd moments is not part of the egress.

The characteristic shape and phases of the eclipse are independent of the photometric band. Over a broad spectral range, from $U$ until $I$, the eclipse seems to be almost grey (MG).

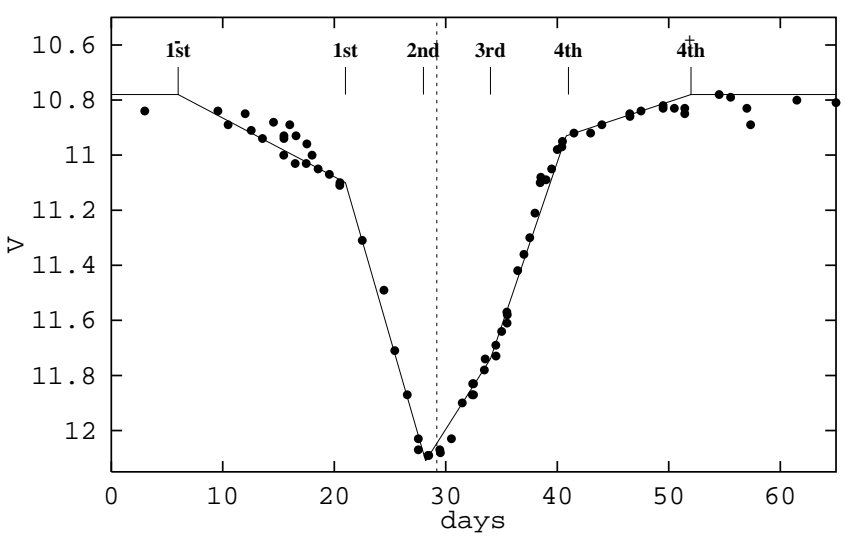

Fig. 3. The characteristic shape of the EE Cep's minima ( $E=4$ and $E=5$ joined, see for details MG). In general six moments can be distinguished in most of minima. The wings corresponding to semiatmospheric eclipse are apparent between 1st-1 st and 4-4th moments. The "true" eclipse can be defined as the part of the light curve between the 1st and 4th moments, with a sloping-bottom between $2 \mathrm{nd}$ and $3 \mathrm{rd}$ contacts.

During three eclipses $(E=4,5,6)$ the depth in the $B$ band was on average 0.07 mag deeper than in the $V$ band, possibly due to a selective extinction of the eclipsing body matter.

\subsection{Depth-duration relation}

Table 1 presents the total duration and the depth of EE Cep's eclipses estimated from data in Figs. 1 and 2. Figure 4 presents the relation between the duration and the depth of eclipses in $B / m_{\mathrm{pg}}$. For epochs 6 and 7 we have only $V /$ vis estimates so we applied a correction of $\Delta m=+0.07$ mag to the depth of these two eclipses. It is worth noting that there is a quite distinct correlation between these two parameters - longer eclipses correspond to shallower minima. Also, from the inspection of Figs. 1 and 2 one can note that the shallowest minima have the most asymmetric wings. The minimum of epoch 7 seems to be an exception to the rule. However, the estimates of $\Delta P$ and $\Delta V$ for this eclipse are based mainly on inconsistent visual 


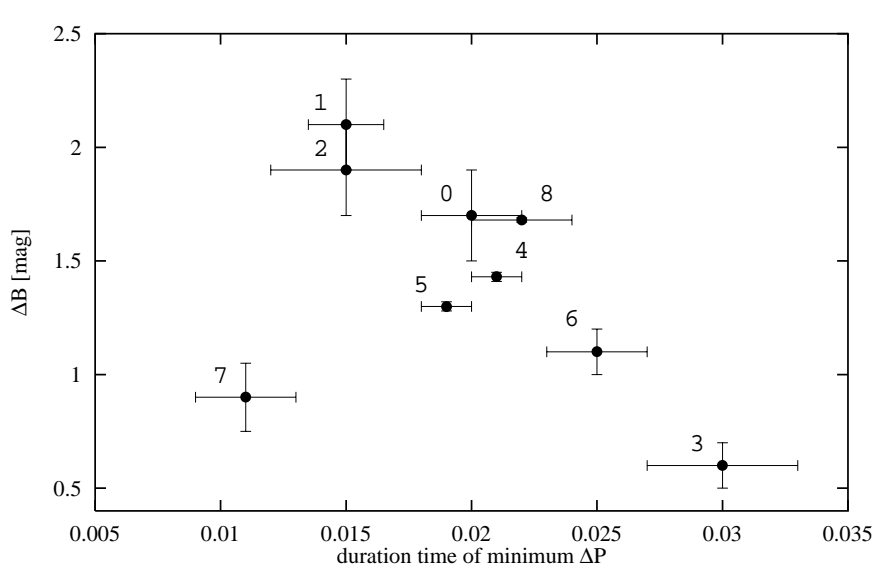

Fig. 4. The depth-duration relation for EE Cep's eclipses. Each eclipse is signed by its epoch number.

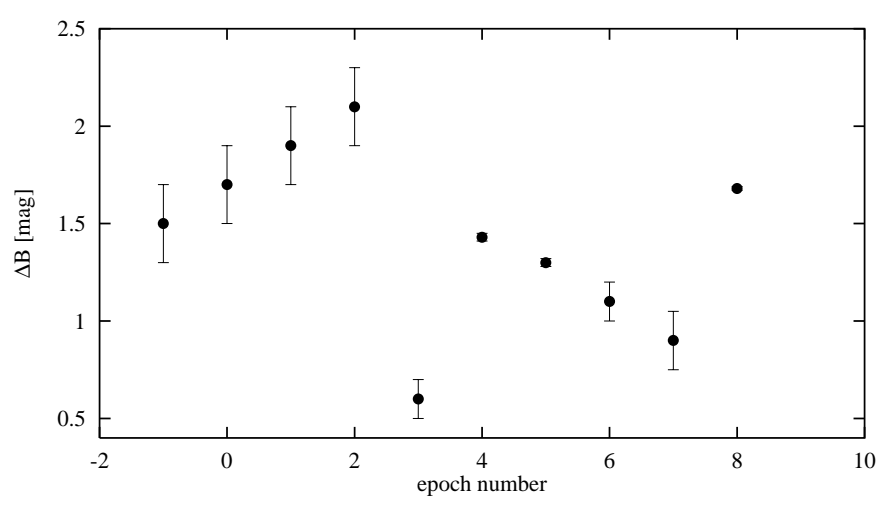

Fig. 5. Time dependence relation for the depth of the eclipses.

observations, this may reflect the presence of a more complex relation than simple linear dependence.

The value of $\Delta B / m_{\mathrm{pg}}$ depths versus the number of epoch is presented in Fig. 5. Its evolution is rather complex, but a possible period of nine epochs ( $\sim 50$ years) can be suggested. Because it is almost equal to the timespan of all the historical data, new observations are needed to confirm its existence. The duration of the eclipses, which is a more difficult parameter to measure, do not show such clear time evolution.

\section{The model of disc eclipses}

The model presented by MG assumes that the invisible component is an optically thick, dark disc inclined to the orbital plane - a "cousin" of the disc in $\epsilon$ Aur system (e.g. Carroll et al. 1991). The spin axis precession of the disc changes two angles: 1) inclination of the disc to the line of sight (which causes changes of the projected area of the disc) and 2) inclination of the projected disc to the orbital movement direction (tilt). As a result we observe varying depth of the minima as it is shown in Fig. 4 in MG. Another kind of a precession - the nodal regression - was recently proposed also for two relatively close eclipsing binaries: SS Lac (Torres 2001) and V907 Sco (Lacy et al. 1999) as a explanation of a varying eclipse depth. In both cases there are precession motion (timescale of hundred years) of the whole short-period (several days) binary orbit caused by an interaction with a third body moving on a wider orbit with a
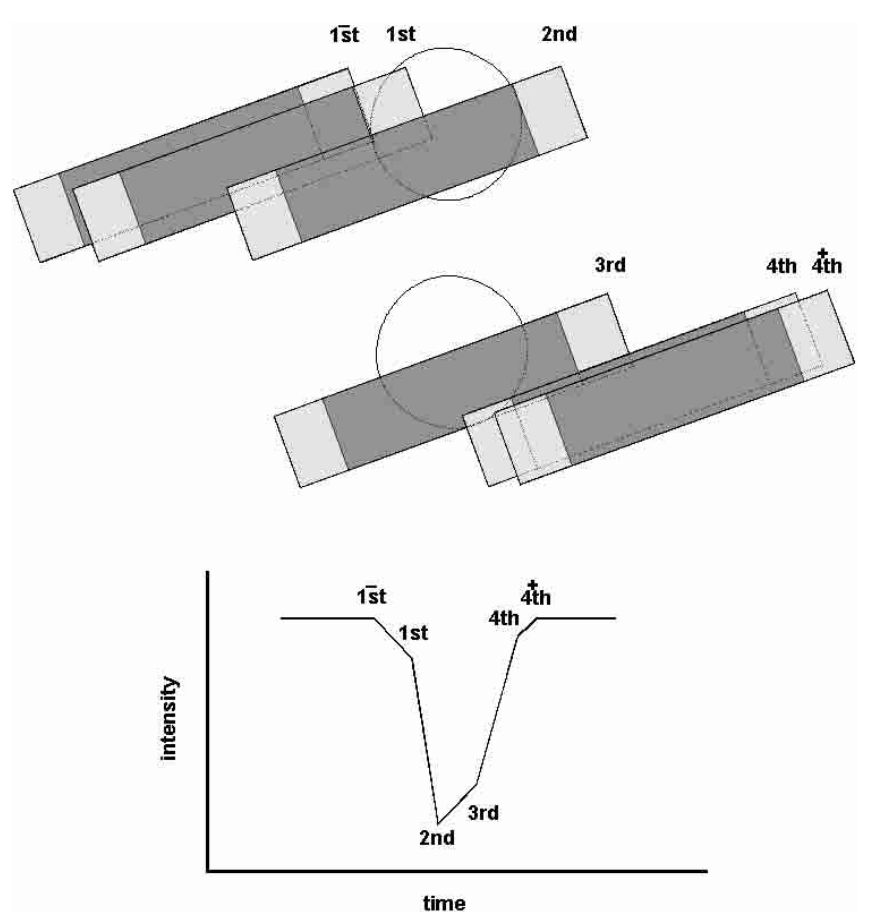

Fig. 6. The schematic explanation of the characteristic moments observed in the light curve of EE Cep during minima via the disc model. An "edge-on" position for the disc was assumed for clarity. The B5 primary is marked by a large circle and semitransparent parts of the disc by a light shadow. Because of the disc tilt during the egress the wing may be much shorter or even unobserved - like during eclipse of epoch 8.

period of hundreds days. In the case of the long period binary EE Cep, however, we observe only the precession of the disc itself.

During our quantitative analysis and after doing some simple numerical tests, we have found that the most probable reason for the observed asymmetries was not the asymmetry of the disc itself but the tilt of the projected disc to the direction of motion. The tilt produces typical asymmetry of the minima as the result of the sloping-bottom between inner contacts (Fig. 6). The wide wings between 1st and 1st, and between 4th and $4^{+}$th may be caused by the outer, semitransparent part of the disc. The "true" eclipse is caused by the opaque, inner part: the four characteristic moments $1 \mathrm{st}-4$ th may be easily understood as contacts between the outer and inner parts of the tilted disc with the eclipsed star - see Figs. 3 and 6. During the extreme, rare, edge-on and small tilt configuration the eclipses should have a flat bottom phase as was observed in 1969. The possible period of nine epochs ( $~ 50$ years) may reflect precession of the disc.

\section{Spectroscopic behaviour}

There are very few spectroscopic observations of EE Cep described in the literature. Herbig (1960) gave its spectral type as $\mathrm{B} 5: \mathrm{ne} \beta$. Brückner (1976) noted that the emission profile in $\mathrm{H}_{\alpha}$ is not typical of Be stars and during the eclipse of epoch 4 there was a considerable strengthening of this emission in respect to the continuum. However, his observations were done 


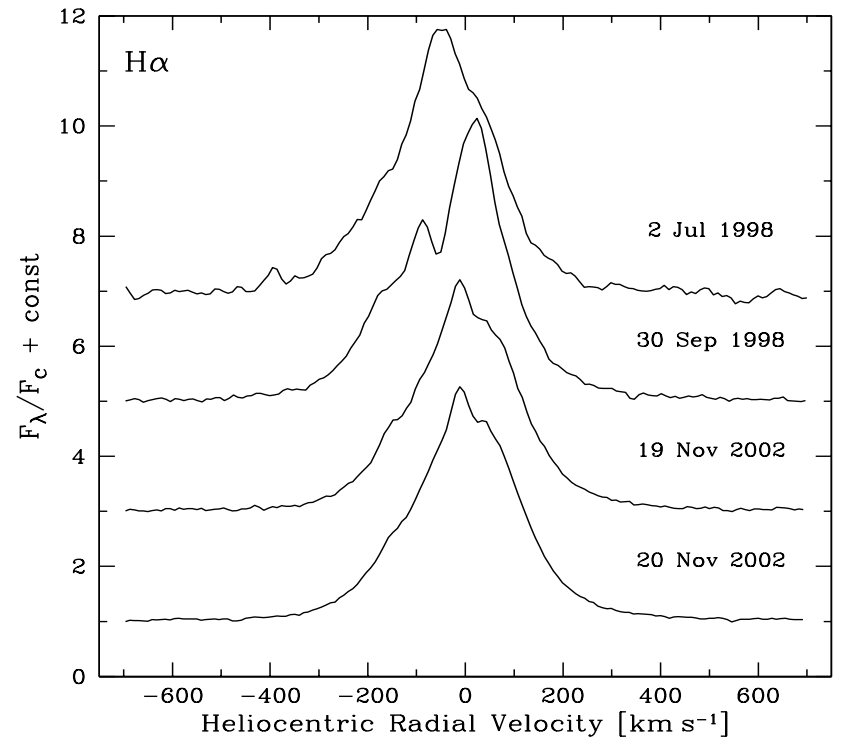

Fig. 7. $\mathrm{H}_{\alpha}$ profile variations in EE Cep spectra obtained outside eclipse.

in very low resolution (200 $\AA / \mathrm{mm})$. Baldinelli et al. (1981) did not record any changes in the spectrum during the following eclipse $(E=5)$, in spite of the slightly higher resolution $(60 \AA / \mathrm{mm})$. Moreover, they noted broad absorption in the Balmer series from $\mathrm{H}_{\alpha}$ to $H_{8}$ with strong, broad $\left(\mathrm{H}_{\alpha}\right)$ and narrow $\left(\mathrm{H}_{\beta}\right)$ emission lines superimposed. This spectrum was estimated as a typical Be star of B5 III spectral type, which agrees with the $B-V, U-B$ colours analysis (MG). Thus, the connection between Balmer emission and eclipsed (Be star) or eclipsing (disc) body is not clear.

We have secured several spectra covering the $\mathrm{H}_{\alpha}$ and the sodium doublet $\mathrm{Na}$ I regions of EE Cep. The spectra were taken at NAO Rozen in Bulgaria on a 2-m telescope equipped with a coude-spectrograph + LN2-cooled CCD camera at resolution $0.4 \AA$. Unexpected large changes of the $\mathrm{H}_{\alpha}$ profile can be noted outside the eclipse phases on a time scale of several months to years (Fig. 7). The $\mathrm{H}_{\alpha}$ profiles are strong and broad $\left(\sim 600 \mathrm{~km} \mathrm{~s}^{-1}\right)$, show one or two peaked emission with variable asymmetries. Two profiles obtained during two consecutive days are almost identical, however a red-shifted bulge around $60 \mathrm{~km} \mathrm{~s}^{-1}$ seems to be stronger in the later spectrum. The sodium doublet $\mathrm{D}_{1} \mathrm{D}_{2}$ shows strong single-component, probably interstellar absorptions. However, its heliocentric radial velocity probably changed from $V_{\mathrm{r}}=-36 \mathrm{~km} \mathrm{~s}^{-1}$ in September 1998 to $V_{\mathrm{r}}=-24 \mathrm{~km} \mathrm{~s}^{-1}$ in November 2002. Also the equivalent width of $\mathrm{D}_{1} \mathrm{D}_{2}$ doublet seems to be variable, decreasing about $10 \%$ between September 1998 and November 2002. The mean value of the $\mathrm{D}_{1}$ equivalent width $E W=0.57 \AA$ corresponds to $E_{B-V}=0.46$ using the calibration given by Munari \& Zwitter (1997), which is fairly consistent with the reddening derived from $U B V$ photometry (MG).

\section{The next eclipse: $2003(E=9)$}

The moment of the mid-eclipse according to the elements given in MG is JD 2452794 (3 June 2003). As shown in Fig. 4 the eclipse can start even about 30 days before mid-eclipse. The reason for it is the presence of the wide wing during ingress which can be usually observed, thus the photometric observations should start at least 40 days before the expected mideclipse. The brightness of the star should decrease by at least 1 mag in $B, V$ filters, but the observers should keep in mind that this decrease may be much larger: up to 2 mag! Also, a significantly shallower (as epochs 3 and 7) minimum can occur. If the periodicity of nine epochs suggested by Fig. 5 is real we can expect an eclipse similar to that of $E=0$, i.e. narrow and deep. Near-far infrared photometry during the eclipses will be very important as it would give the answer to what is the temperature of the companion. Also, good quality $B, V$ or $R$ light curves from the whole eclipse will be valuable to make numerical tests of the disc model.

The spectroscopic observations appear to be very important. We should expect the discovery of a shell spectrum produced by the gaseous, semitransparent part of the disc like in $\epsilon$ Aur (Ferluga \& Mangiacapra 1991). Moreover, such observations would give us an additional clue to the answer of the origin of the variable $\mathrm{H}_{\alpha}$ line. The systematic observations would be very useful to obtain the spectroscopic orbit of the visible B5 component.

The eclipsing body in EE Cep system seems to be unique. The confirmation (or rejection) of the disc hypothesis can be attained only by detailed photometric and spectroscopic study of the incoming eclipses.

Acknowledgements. This paper was supported by KBN Grant No. 5 P03D 00320 and BAN/PAN Joint Research Project No.28. We would like to thank: Ms Karolina Wojtkowska for her help in obtaining some rare literature, dr Alojzy Burnicki for his help in translation of some German publications and dr Boud Roukema for the very careful reading of the manuscript and for the English corrections in the text.

\section{References}

Baldinelli, L., \& Ghedini, S. 1977, Mem. Soc. Astr. It., 48, 91 Baldinelli, L., Ghedini, S., \& Tubertini, C. 1975, IBVS, No. 1009 Baldinelli, L., Ferri, A., \& Ghedini, S. 1981, IBVS, No. 1939

Brückner, V. 1976, Mitt. Verän. Sterne, 7, 114

Carroll, S. M., Guinan, E. F., McCook, G. P., \& Donahue, R. A. 1991, ApJ, 367, 278

Di Luca, R. 1988, G. Assoc. Astrof. Bologna, 92, 5

Ferluga, S., \& Mangiacapra, D. 1991, A\&A, 243, 230

Halbach, E. A. 1992, J. AAVSO, 21, 129

Halbach, E. A. 1999, J. AAVSO, 27, 35

Herbig, G. H. 1960, ApJ, 131, 632

Lacy, C. H. S., Helt, B. E., \& Vaz, L. P. R. 1999, AJ, 117, 541

Meinunger, L. 1973, Mitt. Verän. Sterne, 6, 89

Meinunger, L. 1976, Mitt. Verän. Sterne, 7, 97

Meinunger, L., \& Pfau, A. 1981, Mitt. Verän. Sterne, 9, 38

Mikołajewski, M., \& Graczyk, D. 1999, MNRAS, 303, 521 (MG)

Munari, U., \& Zwitter, T. 1997, A\&A, 318, 269

Perova, N. B. 1957, Peremennye Zvezdy, 12, 125

Romano, G. 1956, Coelum, 24, 135

Romano, G., \& Perissinotto, M. 1966, Mem. Soc. Astr. Ital., 37, 255

Torres, G. 2001, AJ, 121, 2227

Weber, R. 1956, Documentation des Observateurs Circulaires, 9

Zaitseva, G. V., Lyuty, V. M., Perova, N. B., \& Sharov, A. S. 1975, Astron. Cirkular, No. 878, 1 